\title{
VARIABILIDADE ESPACIAL DOS ATRIBUTOS FÍSICOS EM ÁREAS CULTIVADAS COM MAMÃO SOB DIFERENTES PREPAROS DO SOLO
}

\author{
Edney Leandro da Vitória ${ }^{1}$, Deangelys Petene Calvi ${ }^{2}$, Marina Almeida Barbosa ${ }^{3}$, Luciano Canal ${ }^{4}$
}

\section{RESUMO}

O objetivo deste trabalho foi estudar a variabilidade espacial dos atributos físicos do solo em áreas cultivadas com mamoeiro em diferentes preparos de solo, usando as técnicas da estatística clássica e da geoestatística. O experimento foi realizado no ano de 2013, num pomar de mamoeiro Tainung 01, localizado no município de Linhares-ES, em um Argissolo Amarelo Coeso, relevo plano. Os preparos do solo para o transplantio foram feitos em duas áreas adjacentes de aproximadamente 2 ha cada uma, em uma das áreas usou-se duas passadas de uma grade aradora e na outra área duas passadas de um escarificador. Os atributos físicos do solo estudados 90 dias após o transplantio foram a densidade do solo, macroporosidade e microporosidade, além do teor de água no solo nos dois preparos e em duas diferentes faixas de profundidade. Foram selecionadas, aleatoriamente, 100 plantas para formação da malha experimental. Os atributos físicos do solo apresentaram variabilidade espacial com razão de dependência forte para todas variáveis, preparos do solo e profundidade. Os semivarigramas ajustaram-se ao modelo esférico e exponencial para os atributos físicos analisados. De modo geral, observou-se aumento do alcance com o aumento da faixa de profundidade nos dois tipos de preparo do solo, exceto para o teor de água no solo que apresentou uma diminuição dos valores em torno de $23 \%$, tanto no processo de gradagem, quanto na escarificação.

Palavras-chave: geostatística, Carica papaya L. , manejo do solo, propriedades físicas

\section{ABSTRACT \\ SPATIAL VARIABILITY OF PHYSICAL ATTRIBUTES IN AREAS CULTIVATED WITH PAPAYA IN DIFFERENT SOIL TILLAGE}

The objective of this work was to study the spatial variability of soil physical attributes in areas cultivated with papaya subject to different soil tillage, using the techniques of classical statistics and geostatistics. The experiment was carried in 2013 in an orchard of papaya Tainung 01, located in Linhares - ES, on a Ultisol Cohesive, plan relief. The preparation of the soil for transplanting was performed in two adjacent areas of approximately 2 ha each, in one of the areas two passes of a harrow disk were used and in the other area treatment consisted of two passes of a chisel plow. The physical attributes of the soil studied 90 days after transplanting were soil bulk density (DS), macroporosity (Macro) and microporosity (Micro), as well as the water content in the soil in the two preparations and two different depth ranges. To make up the experimental design 100 plants were randomly selected. The soil physical properties showed spatial variability due to strong dependence for all variables, tillage and soil depth. The semi-varigram fit to the spherical and exponential model for the physical attributes analyzed. An increase in the reach $\left(\mathrm{A}_{0}\right)$ was generally observed with increasing depth in both types of tillage, except when the water content in soil showed a decrease in values to roughly $23 \%$, for both harrowing and plowing.

Keywords: geostatistics, Carica papaya, tillage systems, soil physics

\section{Recebido para publicação em 17/12/2013. Aprovado em 03/04/2014.}

1 - Engenheiro Agrícola, Professor, UFES, Departamento de Ciências Agrárias e Biológicas (DCAB/UFES), São Mateus-ES, Brasil.edney.vitoria@ufes.br

2 - Engenheiro Agrônomo, Mestrando, UFES, Programa de Pós-graduação em Agricultura Tropical, São Mateus-ES.

3 - Farmacêutica, Mestranda, UFES, Programa de Pós-graduação em Agricultura Tropical, São Mateus-ES.

4 - Engenheiro Agrônomo, Mestrando, UFES, Programa de Pós-graduação em Agricultura Tropical, São Mateus-ES.

150 REVENG $150-161 \mathrm{p}$.

ENGENHARIA NA AGRICULTURA, VIÇOSA - MG, V.22 N.2, MARÇO / ABRIL 2014 


\section{INTRODUÇÃO}

O setor de fruticultura tem experimentado um aumento crescente nas duas últimas décadas e a globalização do comércio de frutas é uma realidade nos dias atuais. $\mathrm{O}$ aumento das exigências de produtos de qualidade em função do aumento do consumo tornou o mercado frutícola um dos mais competitivos. O Brasil é o primeiro produtor mundial de mamão, com uma produção anual de 1.650.000 toneladas por ano, situando-se entre os principais países exportadores, principalmente para o mercado europeu (ZUFFO, 2012).

Os Estados da Bahia e Espírito Santo são os maiores produtores de mamão do Brasil e possuem uma agricultura relativamente recente quando comparadas às regiões sul e sudeste, e a maioria das práticas agrícolas adotadas nestes Estados foram importadas destas regiões. O preparo e o manejo do solo não são exceção.

Devido ao restrito crescimento de raízes do mamoeiro, a sua sensibilidade a condições de hipoxia do solo e às características dos solos utilizados, o preparo deve ser efetuado para proporcionar boas condições de aeração e drenagem eficiente (COELHO et al., 2008).

Costa et al. (2003) relatam haver poucos resultados de pesquisa que avaliaram o método mais adequado de preparo do solo para o mamoeiro em solos de Tabuleiros Costeiros. Estudo recente, realizado por Zuffo et al. (2013), mostrou que não se justifica o uso de camalhão sobre a linha de plantio confeccionada após o transplantio em relação aos atributos físicos do solo, desenvolvimento sistema radicular e produtividade do mamoeiro.

Os atributos físicos do solo sofrem alterações à medida que são submetidos, sucessivamente, ao uso agrícola (SILVA et al., 2011), tais alterações comprometem o desenvolvimento vegetativo e podem se manifestar de várias formas, influenciando o desenvolvimento das plantas (MARTELLETO $e t$ al., 2008).

Segundo Vieira (2000), a variabilidade dos atributos do solo devem ser consideradas sempre que uma amostragem de campo for efetuada, principalmente quando houver necessidade de um tratamento diferenciado quanto ao manejo, sem qualquer prejuízo para a representatividade, possibilitando maior detalhamento da área. A geoestatística é uma ferramenta eficaz para o estudo da variabilidade espacial dos solos. Para isto, é conveniente que seja feita uma amostragem representativa da área, para que se possa obter dados com valores que, realmente, correspondam à realidade da parcela.

Estudos concluíram que a variabilidade da densidade do solo, resistência do solo à penetração e o teor de água no solo, não ocorrem ao acaso, mas apresentam correlação ou dependência espacial (ROQUE et al., 2008; CAVALCANTE et al., 2011). Para diferentes condições de teores de água no solo, distintos comportamentos da variabilidade espacial da resistência do solo à penetração foram observados por Bottega et al. (2011).

A geostatística, por meio de modelagens, possibilita a descrição quantitativa da variabilidade espacial dos atributos do solo e a estimativa não tendenciosa e com variância mínima de valores desses atributos em locais não amostrados (VIEIRA, 2000). Sendo assim, a geoestatística é ferramenta eficiente de suporte à decisão no manejo do solo, da água e das culturas. Portanto, a análise geoestatística pode indicar alternativas de manejo, não só para reduzir os efeitos da variabilidade do solo sobre a produção da cultura, como também aumentar a possibilidade de estimar respostas das culturas a determinadas práticas de manejo.

Neste contexto, este trabalho teve como objetivo estudar a variabilidade espacial dos atributos físicos do solo em áreas cultivadas com mamoeiro em diferentes preparos de solo, usando as técnicas da estatística clássica e da geoestatística.

\section{MATERIAL E MÉTODOS}

O trabalho foi realizado no ano de 2013, em um pomar de mamoeiro Tainung 01, localizado no município de Linhares-ES, latitude $19^{\circ} 23^{\prime} 28^{\prime \prime}$ $\mathrm{S}$, longitude $40^{\circ} 04^{\prime} 20^{\prime} \mathrm{W}$, a $60 \mathrm{~m}$ de altitude, em um Argissolo Amarelo Coeso, relevo plano, cultivado por cinco anos com Brachiaria brizhanta cv. Marandu e nunca corrigido ou fertilizado. O clima da região é do tipo Aw segundo classificação Köppen, com temperatura média anual de $23{ }^{\circ} \mathrm{C}$ e precipitação média anual de $1.000 \mathrm{~mm}$.

Como parte da caracterização da área 
experimental, procedeu-se a determinação da densidade do solo, da macroporosidade, da microporosidade, resistência do solo à penetração e teor de água no solo, além da análise textural do solo nas faixas de profundidade de 0,00 a 0,20 m e 0,20 a $0,40 \mathrm{~m}$, em 20 pontos selecionados aleatoriamente dentro da área experimental, antes da instalação do experimento.

O preparo do solo para o transplantio foi feito em duas parcelas adjacentes de aproximadamente 2 ha cada uma, em uma das parcelas o solo foi preparado com duas passadas de uma grade aradora tipo off set, Marca Baldan, modelo GTCR, largura de trabalho igual a 2,390 m, com 14 discos recortados e diâmetro de 32", e na outra área, duas passadas de um escarificador, marca Baldan, modelo AETH 7/5, com cinco hastes com ponteira parabólica, largura de trabalho1.525 mm. Os implementos foram tracionados por um trator de pneus marca John Deere, modelo 6125E, potência nominal de $125 \mathrm{cv}(91,9 \mathrm{~kW})$, motor John Deere Power tech 4045 H, Tomada de potência eletroeletrônica independente, distância entre eixos de $2.400 \mathrm{~mm}$ e massa de embarque de $4.420 \mathrm{~kg}$.

O material genético de mamoeiro híbrido F1 Tainung 01 foi utilizado neste experimento, transplantando-se três mudas por cova, em espaçamento $3,8 \times 2,1 \mathrm{~m}$, com posterior sexagem, selecionando uma planta hermafrodita ou uma planta feminina. As mudas foram confeccionadas em tubetes de $60 \mathrm{~mL}$ preenchidos com substrato comercial.

A irrigação foi feita com sistema fixo de aspersão de baixa pressão, com emissores trabalhando com pressão de aproximadamente $200 \mathrm{kPa}$ e vazão de aproximadamente $1,8 \mathrm{~m}^{3} \mathrm{~h}^{-1}$. O controle das plantas daninhas nas entrelinhas foi feito com grade niveladora.

As determinações de atributos físicos do solo foram realizadas aos 90 dias após o transplantio. Foram selecionadas, aleatoriamente, 100 plantas para formação da malha experimental. Foram abertas trincheiras a $0,30 \mathrm{~m}$ ao lado de cada planta selecionada no sentido das entrelinhas, onde foram coletadas amostras de solo nas faixas de profundidade de 0,00 a $0,20 \mathrm{~m}$ e 0,20 a 0,40 m, seguindo metodologia da Embrapa (1997). Os atributos físicos do solo estudados foram:
Densidade do solo (DS), macroporosidade (MACRO) e microporosidade (MICRO), além do teor de água no solo.

Os dados foram submetidos à análise exploratória, a partir da qual se obteve a média e a mediana como medidas de tendência central, a variância, desvio padrão e o coeficiente de variação como medidas de dispersão. Também foram determinados os coeficientes de assimetria e curtose para a verificação da posição dos dados em relação à distribuição normal. Realizouse a identificação dos outliers, efetuando a substituição dos seus valores pelo valor médio dos circunvizinhos contidos na malha. Para testar a hipótese de normalidade, ou de lognormalidade, realizou-se o teste de Kolmogorov-smirnov, a 5\% de probabilidade. O software R versão 3.0.2 (2013) foi utilizado nesta análise exploratória dos dados.

A estrutura de dependência espacial foi avaliada por meio da geoestatística calculando o semivarigrama experimental (Equação 1):

$$
\gamma *(h)=1 / 2 N(h) \sum_{i=1}^{N(h)}\left[Z\left(s_{i}\right)-Z\left(s_{i}+h\right)\right]^{2}
$$

em que,

$\gamma^{*}(\mathrm{~h})=$ semivariância experimental obtida pelos valores amostrados;

$\mathrm{h}=$ distância entre os pontos amostrais;

$\mathrm{N}$ (h) é o número de pares de valores medidos $\mathrm{Z}_{1}$ (si), $\mathrm{Z}_{1}(\mathrm{si}+\mathrm{h})$ e $\mathrm{Z}_{2}(\mathrm{si}), \mathrm{Z}_{2}(\mathrm{si}+\mathrm{h})$ para as variáveis $\mathrm{Z}_{1}$ e $\mathrm{Z}_{2}$, separados por uma distância amostral, vetor $\mathrm{h}$ (VIEIRA, 2000; WALTER et al., 2002).

Os ajustes dos semivariogramas, em função de seus modelos, foram efetuados pela seleção inicial de: menor soma dos quadrados dos desvios (SQD); maior coeficiente de determinação $\left(\mathrm{R}^{2}\right)$; e maior avaliador da dependência espacial. A decisão final do modelo que representou o ajuste foi realizada pela validação cruzada, assim como para a definição do tamanho da vizinhança que proporcionou a melhor malha de krigagem. Para cada atributo, foram relacionados o efeito pepita (Co), o alcance (Ao) e o patamar $(\mathrm{Co}+\mathrm{C})$. A análise do avaliador da dependência espacial (ADE) foi efetuada conforme a seguinte expressão:

\section{REVENG}

$$
\text { 150-161p. }
$$

ENGENHARIA NA AgRicultura, VIÇOSA - MG, V.22 N.2, MARÇO / AbRIL 2014 
$A D E=\frac{C}{C+C_{0}} \times 100$

em que,

$\mathrm{ADE}=$ avaliador da dependência espacial;

$\mathrm{C}=$ variância estrutural;

$\mathrm{C}+\mathrm{C}_{0}=$ patamar.

A interpretação proposta para o ADE foi a seguinte: $\mathrm{ADE} \leq 25 \%$ indica variável espacial fracamente dependente; $25 \% \leq \mathrm{ADE} \leq 75 \%$ indica variável espacial moderadamente dependente; e $\mathrm{ADE}>75 \%$ indica variável espacial fortemente dependente.

\section{RESULTADO E DISCUSSÃO}

As frações de areia, silte e argila, obtidas pela granulometria do solo da área experimental são apresentadas no Quadro 1. Os solos com esta textura são típicos de solos de tabuleiros costeiros encontrados no Estado do Espírito Santo, valores similares foram encontrados por Zuffo (2012) em uma avaliação preliminar da textura do solo localizado em uma área do município de Boa Esperança, região Norte do Estado. O solo foi classificado como de textura arenosa, seguindo descrições da Embrapa (1999).

No Quadro 2 são apresentadas propriedades físicas médias do solo na área experimental em duas faixas de profundidade, os maiores valores médios de densidade do solo, resistência do solo a penetração e teor de água no solo foram observados na faixa de 0,20 a 0,40 m. Souza (2013) encontrou resultados similares quando estudou os atributos físico-hídricos do solo no município de São Mateus, região norte do Espírito Santo, em solo com as mesmas características deste estudo.

Quadro 1. Análise textural do solo nas faixas de profundidades estudadas antes dos preparos do solo.

\begin{tabular}{llcc}
\hline \multirow{2}{*}{ Fração } & \multirow{2}{*}{ Unidade } & \multicolumn{2}{c}{ Faixa de profundidade } \\
\cline { 3 - 4 } Areia grossa & $\mathrm{kg} \mathrm{kg}^{-1}$ & $0,00 \mathrm{a} 0,20 \mathrm{~m}$ & $0,20 \mathrm{a} 0,40 \mathrm{~m}$ \\
Areia fina & $\mathrm{kg} \mathrm{kg}^{-1}$ & 0,173 & 0,439 \\
Areia total & $\mathrm{kg} \mathrm{kg}^{-1}$ & 0,749 & 0,188 \\
Silte & $\mathrm{kg} \mathrm{kg}^{-1}$ & 0,126 & 0,627 \\
Argila & $\mathrm{kg} \mathrm{kg}^{-1}$ & 0,136 & 0,120 \\
\hline
\end{tabular}

Quadro 2. Valores médios \pm desvio padrão da densidade do solo $\left(\mathrm{g} \mathrm{cm}^{-2}\right)$, macroporosidade $\left(\mathrm{m}^{3} \mathrm{~m}^{-3}\right)$, microposrosidade $\left(\mathrm{m}^{3} \mathrm{~m}^{-3}\right)$, resistência do solo a penetração (MPa) e teor de água no solo $(\mathrm{kg}$ $\mathrm{kg}^{-1}$ ) nas faixas de profundidade estudadas antes dos preparos do solo.

\begin{tabular}{lcc}
\hline & \multicolumn{2}{c}{ Faixa de profundidade } \\
\cline { 2 - 3 } Atributo & 0,00 a $0,20 \mathrm{~m}$ & 0,20 a $0,40 \mathrm{~m}$ \\
\hline Densidade do solo & $1,59 \pm 0,05$ & $1,63 \pm 0,04$ \\
Macroporosidade & $0,18 \pm 0,03$ & $0,13 \pm 0,01$ \\
Microporosidade & $0,19 \pm 0,02$ & $0,22 \pm 0,01$ \\
Resistência a penetração & $2,26 \pm 1,89$ & $5,05 \pm 1,56$ \\
Teor de água no solo & $6,60 \pm 0,30$ & $7,31 \pm 0,60$ \\
\hline
\end{tabular}


No Quadro 3 são apresentados os dados relativos a análise descritiva dos atributos físicos do solo. Os valores de média e mediana são próximos para todos os atributos nas faixas de profundidade de 0,00 a $0,20 \mathrm{~m}$ e 0,20 a $0,40 \mathrm{~m}$, indicando a distribuição simétrica para os dados e a normalidade pelo teste de Kolmogorov-smirnov, em nível de 5\% de significância.

Analisando o Quadro 3, verifica-se que os coeficientes de assimetria e curtose para a maioria dos atributos são próximos de zero, ou seja, compatíveis com a distribuição normal. Porém, os atributos como a microporosidade, em ambas as faixas de profundidade e preparo do solo, e o teor de água no solo na escarificação do solo e profundidade de 0,00 a $0,20 \mathrm{~m}$ não apresentaram distribuição normal. Este fato pode estar relacionado com seu processo de determinação no laboratório, ou da variabilidade dos atributos em condição de campo. De acordo com Silva (2012), tais comportamentos são típicos de solos de tabuleiros costeiros.

A variabilidade dos atributos do solo pode ser constatada pelo coeficiente de variação $(\mathrm{CV})$, segundo a classificação proposta por Warrick e Nielsen (1980). A densidade do solo e o teor de água no solo apresentaram os menores valores de $\mathrm{CV}$, sendo considerados baixos $(\mathrm{CV}<6 \%)$. O teor de água no solo na gradagem e faixa de profundidade de 0,00 a $0,20 \mathrm{~m}$ apresentou o menor valor de CV, igual $2,4 \%$.

Quadro 3. Estatística descritiva dos dados de densidade do solo $\left(\mathrm{g} \mathrm{cm}^{-2}\right)$, teor de água no solo $(\mathrm{kg}$ $\left.\mathrm{kg}^{-1}\right)$, macroporosidade $\left(\mathrm{m}^{3} \mathrm{~m}^{-3}\right)$, microposrosidade $\left(\mathrm{m}^{3} \mathrm{~m}^{-3}\right)$ e relação entre macro $\mathrm{e}$ microporosidade nas faixas de profundidade 0,00 a $0,20 \mathrm{~m}$ e 0,20 a $0,40 \mathrm{~m}$ nos dois sistemas de preparo do solo.

\begin{tabular}{|c|c|c|c|c|c|c|c|c|}
\hline \multicolumn{9}{|c|}{ Gradagem $(0,00$ a $0,20 \mathrm{~m})$} \\
\hline Variável & Média & Mediana & $\mathrm{s}^{2}$ & $\mathrm{~s}$ & $\mathrm{CV}$ & $\mathrm{C}_{\mathrm{s}}$ & $\mathrm{C}_{\mathrm{k}}$ & $\mathrm{d}$ \\
\hline DS & 1,28 & 1,29 & 0,004 & 0,07 & 5,1 & 0,10 & 0,51 & $0,061^{\mathrm{ns}}$ \\
\hline$\Theta$ & 12,86 & 12,84 & 0,097 & 0,31 & 2,4 & 0,29 & 0,55 & $0,101^{\mathrm{ns}}$ \\
\hline Macro & 0,321 & 0,322 & 0,007 & 0,09 & 26,5 & 0,09 & $-0,55$ & $0,080^{\mathrm{ns}}$ \\
\hline Micro & 0,151 & 0,164 & 0,001 & 0,04 & 24,8 & $-0,18$ & 0,03 & $0,044 *$ \\
\hline \multicolumn{9}{|c|}{ Gradagem $(0,20$ a $0,40 \mathrm{~m})$} \\
\hline DS & 1,55 & 1,54 & 0,002 & 0,04 & 2,6 & 0,02 & 0,29 & $0,072^{\mathrm{ns}}$ \\
\hline$\Theta$ & 12,41 & 12,33 & 0,098 & 0,31 & 2,5 & 0,22 & 0,06 & $0,081^{\mathrm{ns}}$ \\
\hline Macro & 0,151 & 0,162 & 0,002 & 0,05 & 32,2 & $-0,43$ & 0,22 & $0,082^{\mathrm{ns}}$ \\
\hline Micro & 0,213 & 0,200 & 0,004 & 0,07 & 31,3 & $-0,24$ & $-0,03$ & $0,024^{*}$ \\
\hline \multicolumn{9}{|c|}{ Escarificação $(0,00$ a 0,20 m) } \\
\hline DS & 1,35 & 1,34 & 0,003 & 0,05 & 3,9 & 0,61 & 0,06 & $0,087^{\text {ns }}$ \\
\hline$\Theta$ & 12,71 & 12,62 & 0,558 & 0,75 & 5,9 & $-0,20$ & 0,13 & $0,039^{*}$ \\
\hline Macro & 0,302 & 0,313 & 0,008 & 0,09 & 31,1 & $-0,52$ & 0,50 & $0,054^{\text {ns }}$ \\
\hline Micro & 0,144 & 0,135 & 0,002 & 0,04 & 30,4 & $-0,26$ &,- 014 & $0,040^{*}$ \\
\hline \multicolumn{9}{|c|}{ Escarificação $(0,20$ a 0,40 m) } \\
\hline DS & 1,43 & 1,42 & 0,003 & 0,06 & 4,2 & 0,11 & $-0,26$ & $0,087^{\mathrm{ns}}$ \\
\hline$\Theta$ & 13,47 & 13,46 & 0,204 & 0,45 & 3,4 & 0,07 & $-0,36$ & $0,069^{\text {ns }}$ \\
\hline Macro & 0214 & 0,213 & 0,006 & 0,08 & 37,2 & 0,06 & $-0,09$ & $0,054^{\text {ns }}$ \\
\hline Micro & 0,170 & 0,169 & 0,003 & 0,06 & 32,4 & 0,14 & $-0,22$ & $0,040^{*}$ \\
\hline
\end{tabular}

$\mathrm{s}^{2}=$ variância; $\mathrm{s}=$ desvio padrão; $\mathrm{C}_{\mathrm{s}}=$ coeficiente de assimetria; $\mathrm{C}_{\mathrm{k}}=$ coeficiente de curtose; $\mathrm{d}=$ nível de significância do teste de Kolmogorov-smirnov. 
A macroporosidade e a microporosidade apresentaram valor de CV entre 24,8 e $37,2 \%$, classificados como moderados (12\% a $60 \%)$, o maior valor de CV foi observado para macroporosidade na escarificação e faixa de profundidade de 0,20 a $0,40 \mathrm{~m}$, igual a $37,2 \%$. Tavares et al. (2012), estudando a relação espacial de atributos físicos e mecânicos de um Argissolo Coeso, observaram comportamento semelhante em relação ao coeficiente de variação para as variáveis estudadas.

Ainda que os valores de $\mathrm{CV}$ observados de macro e microporosidade sejam moderados, este não é bom indicador da variabilidade espacial de atributos do solo, pois em situação de campo pode ocorrer valores altos ou baixos (SANCHEZ et al., 2009).

Zucoloto et al. (2011) estudaram a variabilidade espacial de atributos físicos do solo e da produtividade em um pomar de laranjas em um Latossolo Amarelo Distrófico e concluíram que os coeficientes de variação para a porosidade total e densidade do solo apresentaram variabilidade baixa. Portanto, o emprego do $\mathrm{CV}$ não deve ser generalizado, devendo apreciar estes resultados segundo as finalidades a que se destina o trabalho.

$\mathrm{Na}$ análise geoestatística realizada nos dados, por meio de semivariogramas (Quadro 4), os atributos físicos do solo estudado apresentaram dependência espacial, dado que nenhum apresentou efeito pepita puro, fato que poderia determinar uma distribuição aleatória dos dados. Observa-se que os semivariogramas ajustaram-se ao modelo esférico e exponencial para os atributos físicos analisados. Sanchez et al. (2009) realizaram um experimento para caracterizar a variabilidade espacial de atributos de um solo Argissolo Vermelho-amarelo e encontraram semivarigramas exponenciais e esféricos. O modelo esférico é considerado por muitos autores como sendo o que melhor se ajusta aos atributos físicos do solo (FONTENELE et al., 2009; CORRÊA et al., 2010; MELO, 2012).

Analisando o Quadro 4 e a Figura 1, observa-se que a faixa de dependência espacial, definida pelo alcance $\left(\mathrm{A}_{0}\right)$, no atributo de densidade do solo foi encontrado após o processo de escarificação, na faixa de profundidade de 0,20 a $0,40 \mathrm{~m}$, no valor de $21,2 \mathrm{~m}$. O maior $\mathrm{A}_{0}$ para o teor de água no solo foi de $26,1 \mathrm{~m}$, após gradagem, na faixa de profundidade de 0,00 a $0,20 \mathrm{~m}$, e para os atributos macroporosidade e microporosidade os maiores valores de $\mathrm{A}_{0}$ foram 36,1 e $38,1 \mathrm{~m}$, respectivamente, após o processo de gradagem e na faixa de profundidade de 0,20 a $0,40 \mathrm{~m}$. De modo geral, observou-se aumento do $\mathrm{A}_{0}$ com o aumento da faixa de profundidade nos dois tipos de preparo do solo, exceto para o teor de água no solo que apresentou uma diminuição dos valores em torno de $23 \%$, tanto no processo de gradagem, quanto na escarificação. À medida que a profundidade aumenta a variabilidade do teor de água no solo diminuiu fazendo que o alcance seja menor.

Soares (2013), analisando os atributos físicos e químicos do solo sob diferentes sistemas agrícolas no municipio de Apodi - RN, em solo semelhante ao estudado, constatou diminuição do teor de água no solo em função da profundidade, corroborando com os resultados encontrados.

Zuffo et al. (2013) estudaram os efeitos de diferentes preparos de solo nos atributos físicos do solo e no desempenho produtivo do mamoeiro na região norte do Espírito Santo e constataram que o preparo convencional e cultivo mínimo, seguidos ou não de subsolagem, não incrementam positivamente nos atributos de densidade do solo, macroporosidade, microporosidade e teor de água no solo, tais resultados reforçam o valor do alcance encontrado, dado que observou-se uma grande variabilidade dos resultados.

A interpretação correta dos resultados dos alcances encontrados dos atributos físicos estudados é importante no planejamento da área experimental, portanto, tais resultados devem ser levados em consideração nas propostas de manejo e pesquisa, assim como o número de pontos que devem ser amostrados para aumentar a representatividade (VITÓRIA et al., 2012).

$\mathrm{O}$ grau de dependência espacial (ADE) em todos os atributos físicos do solo, preparos do solo e faixas de profundidade foram consideradas altas, pois foram superiores a $75 \%$. Este resultado pode ser explicado dado ao fato de que as variáveis que apresentam forte grau de dependência são mais influenciadas por propriedades intrínsecas do solo, tais como textura, estrutura, teor de matéria orgânica, densidade, mineralogia e conteúdo de água (CARNEIRO et al., 2009; VENDRUSCOLO et al., 2011; SOARES (2013). Tavares et al. (2012), analisando a relação espacial da densidade do solo, porosidade, macroporosidade, microporosidade e teor de água no solo, verificaram grau de dependência espacial de média (entre 25 a $75 \%$ ) a forte (superior a 75\%) para todos os atributos indicando que tais variáveis não aleatórias no espaço. 
Quadro 4. Modelos e parâmetros dos semivariogramas ajustados aos dados de densidade do solo (g $\left.\mathrm{cm}^{-2}\right)$, teor de água no solo $\left(\mathrm{kg} \mathrm{kg}^{-1}\right)$, macroporosidade $\left(\mathrm{m}^{3} \mathrm{~m}^{-3}\right)$, microporosidade $\left(\mathrm{m}^{3} \mathrm{~m}^{-3}\right)$ e relação entre macro e microporosidade nas faixas de profundidade 0,00 a $0,20 \mathrm{~m}$ e 0,20 a $0,40 \mathrm{~m}$ nos dois sistemas de preparo do solo.

\begin{tabular}{|c|c|c|c|c|}
\hline \multicolumn{5}{|c|}{ Gradagem $(0,00$ a $0,20 \mathrm{~m})$} \\
\hline & DS & $\Theta$ & Macro & Micro \\
\hline Modelo & Esférico & Esférico & Esférico & Exponencial \\
\hline $\mathrm{C}_{0}$ & $1,00 \times 10^{-5}$ & $3,82 \times 10^{-3}$ & $5,63 \times 10-4$ & $8,63 \times 10^{-5}$ \\
\hline $\mathrm{C}+\mathrm{C}_{0}$ & $4,21 \times 10^{-3}$ & $1,01 \times 10^{-1}$ & $7,65 \times 10^{-3}$ & $1,47 \times 10^{-3}$ \\
\hline $\mathrm{A}_{0}$ & 18,0 & 26,1 & 30,0 & 31,3 \\
\hline $\mathrm{ADE}$ & Forte & Forte & Forte & Forte \\
\hline $\mathrm{R}^{2}$ & 0,88 & 0,90 & 0,86 & 0,91 \\
\hline SQD & $2,82 \times 10^{-7}$ & $3,37 \times 10^{-7}$ & $1,14 \times 10^{-6}$ & $8,77 \times 10^{-4}$ \\
\hline \multicolumn{5}{|c|}{ Gradagem $(0,20$ a $0,40 \mathrm{~m})$} \\
\hline Modelo & Esférico & Esférico & Exponencial & Exponencial \\
\hline $\mathrm{C}_{0}$ & $5,00 \times 10^{-5}$ & $2,80 \times 10^{-4}$ & $4,00 \times 10^{-3}$ & $2,61 \times 10^{-4}$ \\
\hline $\mathrm{C}+\mathrm{C}_{0}$ & $4,91 \times 10^{-3}$ & $4,10 \times 10^{-3}$ & $5,31 \times 10^{-2}$ & $2,28 \times 10^{-3}$ \\
\hline $\mathrm{A}_{0}$ & 19,7 & 17,8 & 36,1 & 38,1 \\
\hline $\mathrm{ADE}$ & Forte & Fote & Forte & Forte \\
\hline $\mathrm{R}^{2}$ & 0,79 & 0,90 & 0,94 & 0,95 \\
\hline SQD & $4,63 \times 10^{-7}$ & $3,25 \times 10^{-7}$ & $6,32 \times 10^{-6}$ & $4,46 \times 10^{-8}$ \\
\hline \multicolumn{5}{|c|}{ Escarificação $(0,00$ a $0,20 \mathrm{~m})$} \\
\hline Modelo & Esférico & Esférico & Esférico & Esférico \\
\hline $\mathrm{C}_{0}$ & $1,41 \times 10^{-4}$ & $2,40 \times 10^{-2}$ & $5,60 \times 10^{-4}$ & $4,00 \times 10^{-6}$ \\
\hline $\mathrm{C}+\mathrm{C}_{0}$ & $2,92 \times 10^{-3}$ & $5,76 \times 10^{-1}$ & $8,89 \times 10^{-3}$ & $1,74 \times 10-3$ \\
\hline $\mathrm{A}_{0}$ & 16,6 & 24,5 & 24,5 & 28,7 \\
\hline $\mathrm{ADE}$ & Forte & Forte & Forte & Forte \\
\hline $\mathrm{R}^{2}$ & 0,82 & 0,91 & 0,83 & 0,88 \\
\hline SQD & $3,93 \times 10^{-7}$ & $2,71 \times 10^{-6}$ & $2,05 \times 10^{-6}$ & $2,51 \times 10^{-8}$ \\
\hline \multicolumn{5}{|c|}{ Escarificação $(0,20$ a 0,40 m) } \\
\hline Modelo & Esférico & Esférico & Esférico & Exponencial \\
\hline $\mathrm{C}_{0}$ & $1,67 \times 10^{-4}$ & $8,8 \times 10^{-4}$ & $8,40 \times 10^{-3}$ & $1,31 \times 10^{-4}$ \\
\hline $\mathrm{C}+\mathrm{C}_{0}$ & $2,53 \times 10^{-3}$ & $4,8 \times 10^{-3}$ & $2,58 \times 10^{-1}$ & $1,56 \times 10^{-3}$ \\
\hline $\mathrm{A}_{0}$ & 21,2 & 17,4 & 24,6 & 30,0 \\
\hline $\mathrm{ADE}$ & Forte & Forte & Forte & Forte \\
\hline $\mathrm{R}^{2}$ & 0,83 & 0,87 & 0,91 & 0,91 \\
\hline SQD & $1,57 \times 10^{-7}$ & $4,37 \times 10^{-4}$ & $5,40 \times 10^{-8}$ & $2,94 \times 10^{-7}$ \\
\hline
\end{tabular}

Os resultados da análise variográfica mostraram que todos os atributos físicos tiveram dependência espacial (Figuras 1 e 2). Vários modelos foram testados até que um fosse considerado ótimo, conforme, metodologia empregada por Vitória (2010).

Quando a anisotropia ocorre, a direção afeta a estrutura da variabilidade espacial em termos dos parâmetros: efeito pepita; alcance e patamar.
A decisão da estacionariedade, os estimadores utilizados, o modelo do semivariograma ajustado e a decisão sobre a isotropia adotada, ou seja, todo o processo de modelagem foi testado pela validação cruzada (VIEIRA, 2000). Nenhum modelo anisotrópico foi superior aos modelos isotrópicos. Os modelos dos semivariogramas ajustados foram o esférico e o exponencial. 
$\operatorname{DS}(0,00 \mathrm{a} 0,20 \mathrm{~m})$

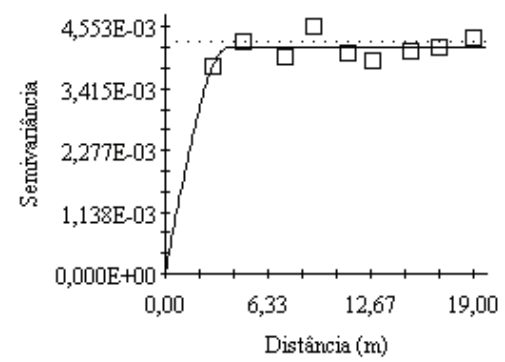

umidade $(0,00 \mathrm{a} 0,20 \mathrm{~m})$

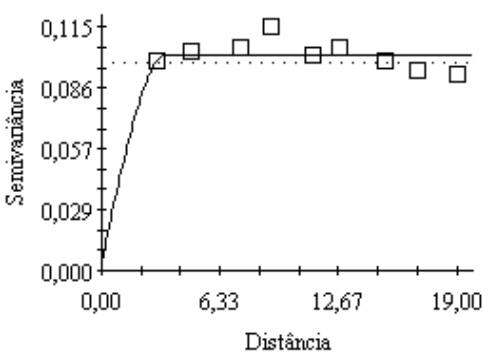

Macro $(0,00$ a $0,20 \mathrm{~m})$

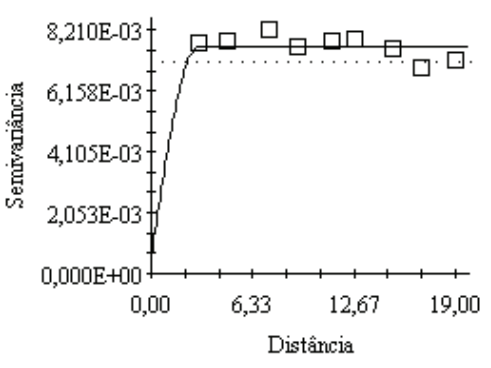

Micro $(0,00$ a $0,20 \mathrm{~m})$

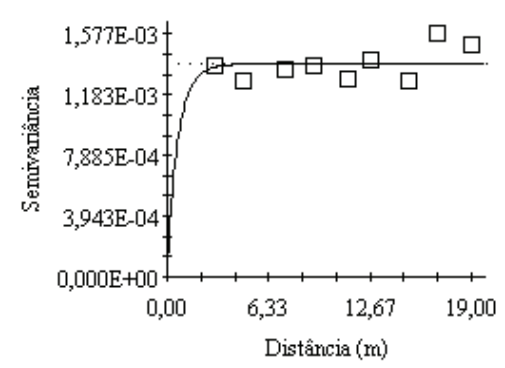

$\operatorname{DS}(0,20 \mathrm{a} 0,40 \mathrm{~m})$

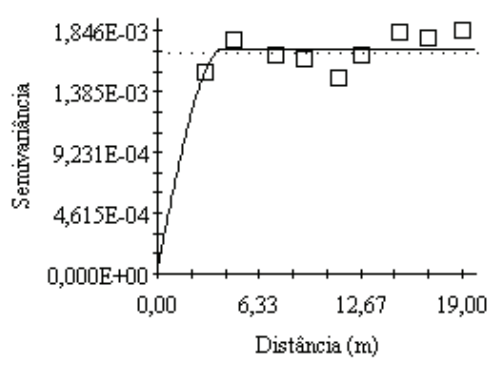

urridade $(0,20$ a $0,40 \mathrm{~m})$

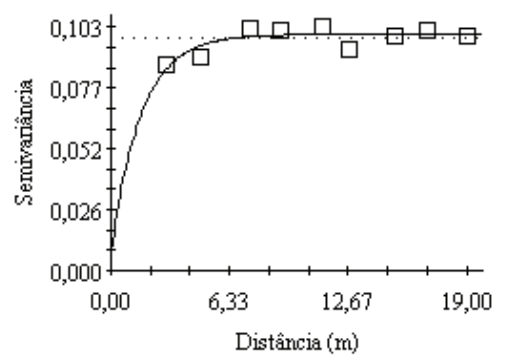

$\operatorname{Macro}(0,20$ a $0,40 \mathrm{~m})$

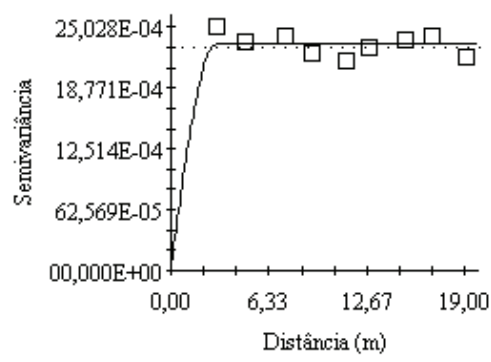

Micro $(0,20$ a $0,40 \mathrm{~m})$

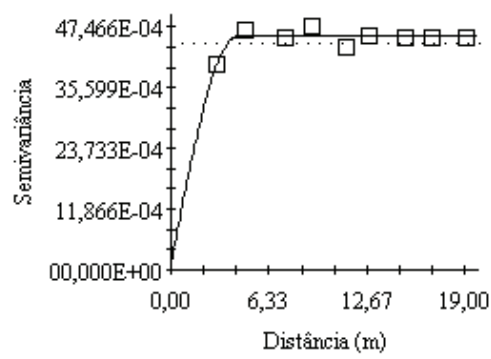

Figura 1. Semivariogramas dos atributos físicos do solo: densidade do solo $\left(\mathrm{g} \mathrm{cm}^{-2}\right)$, teor de água no solo $\left(\mathrm{kg} \mathrm{kg}^{-1}\right)$, macroporosidade $\left(\mathrm{m}^{3} \mathrm{~m}^{-3}\right)$, microporosidade $\left(\mathrm{m}^{3} \mathrm{~m}^{-3}\right)$ e relação entre macro e microporosidade nas faixas de profundidade de 0,00 a $0,20 \mathrm{~m}$ a 0,20 a $0,40 \mathrm{~m}$ na área preparada com grade aradora. 
DS $(0,00$ a $0,20 \mathrm{~m})$

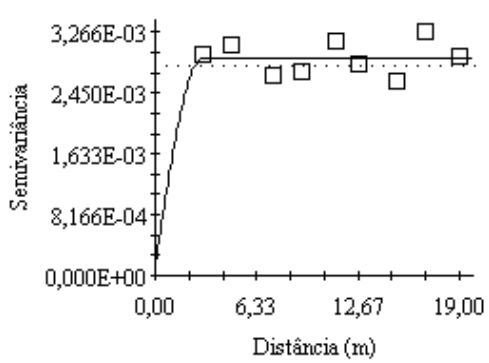

urnidade $(0,00$ a $0,20 \mathrm{~m})$

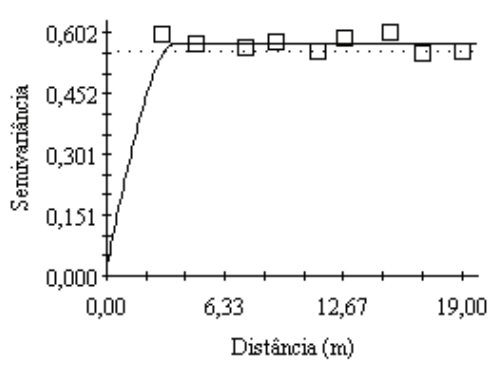

Macro $(0,00$ a 0,20 m)

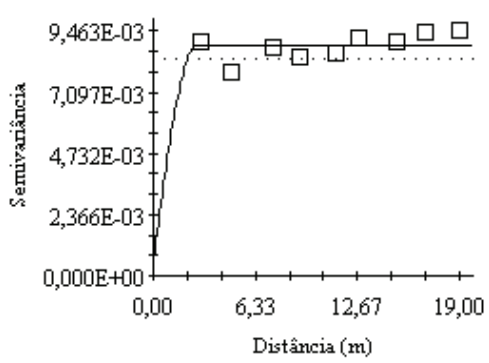

Micro $(0,00$ a 0,20 m)

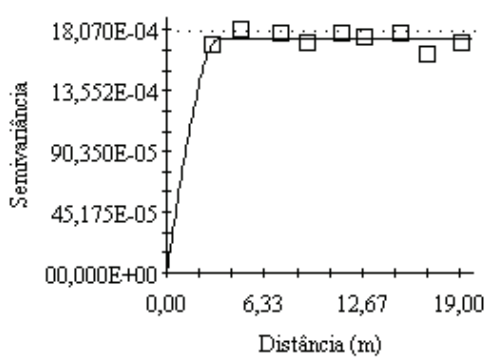

$\operatorname{DS}(0,20 \mathrm{a} 0,40 \mathrm{~m})$

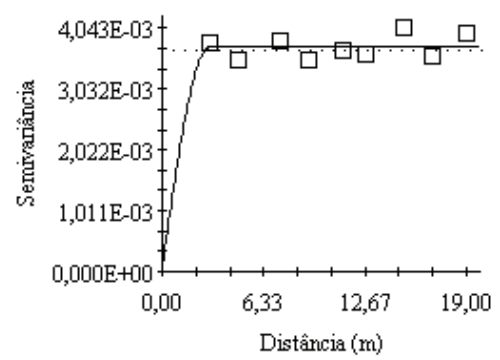

umidade $(0,20$ a $0,40 \mathrm{~m})$

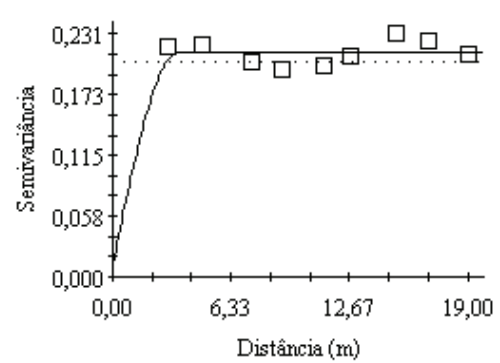

$\operatorname{Macro}(0,20$ a 0,40 m)

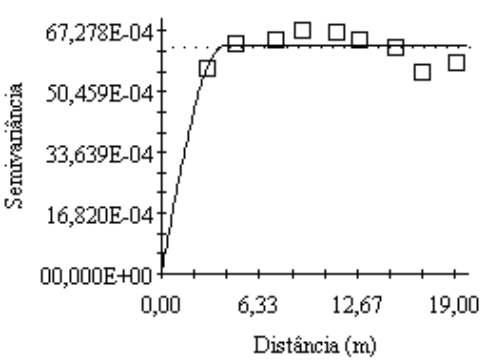

Micro $(0,20$ a 0,40 m)

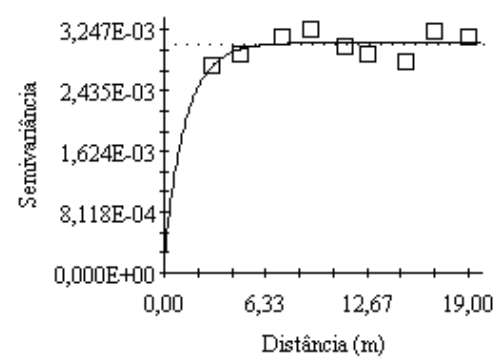

Figura 2. Semivariogramas dos atributos físicos do solo: densidade do solo $\left(\mathrm{g} \mathrm{cm}^{-2}\right)$, teor de água no solo $\left(\mathrm{kg} \mathrm{kg}^{-1}\right)$, macroporosidade $\left(\mathrm{m}^{3} \mathrm{~m}^{-3}\right)$, microporosidade $\left(\mathrm{m}^{3} \mathrm{~m}^{-3}\right)$ e relação entre macro e microporosidade nas faixas de profundidade de 0,00 a $0,20 \mathrm{~m}$ a 0,20 a $0,40 \mathrm{~m}$ na área preparada com escarificador.

\section{REVENG




\section{CONCLUSÕES}

- Os coeficientes de variação indicaram variabilidade baixa para a densidade do solo e teor de água no solo e alta para macroporosidade e microporosidade nos preparos de solo estudados independente da faixa de profundidade;

- Os atributos de solo apresentaram elevado grau de dependência espacial, independente do preparo do solo e faixa de profundidade;

- Os valores de alcance encontrados para os atributos físicos do solo podem ser levados em consideração em planejamento experimental, especificamente na definição da amostragem.

\section{REFERÊNCIAS BIBLIOGRÁFICAS}

BOTTEGA, E.L.; BOTTEGA, S.P.; SILVA, S.A.; QUEIROZ, D.M.; SOUZA, C.M.A.; RAFULL, L.Z.L. Variabilidade espacial da resistência do solo à penetração em um Latossolo Vermelho distroférrico. Revista Brasileira de Ciências Agrárias (Agrária)/ Brazilian Journal of Agricultural Sciences, Recife, v.6, n.2, p.331-336, 2011.

CARNEIRO, M.A.C.; SOUZA, E.D.; REIS, E.D.; PEREIRA, H.S.; AZEVEDO, W.D. Atributos físicos, químicos e biológicos de solo de cerrado sob diferentes sistemas de uso e manejo. Revista Brasileira de Ciência do Solo, Viçosa, v.33, n.1, p.147-157, 2009.

CAVALCANTE, E.G.; ALVES, M.C.; SOUZA, Z.M.; PEREIRA, G. Variabilidade espacial de atributos físicos do solo sob diferentes usos e manejos. Revista Brasileira de Engenharia Agrícola e Ambiental, Campina Grande, v.15, n.1, p.237-243, 2011.

COELHO, E.F.; SIMÕES, W.L. Distribuição de raízes e extração de água em fruteiras tropicais. Cruz das Almas: Embrapa Mandioca e Fruticultura, 2008. 80p.

CORRÊA, R.M.; FREIRE, M.B.D.S.; FERREIRA, R.L.; SILVA, J.A.; PESSOA, L.G.; MIRANDA,
M.A.; MELO, D.V. Atributos físicos de solos sob diferentes usos com irrigação no semiárido de Pernambuco1. Revista Brasileira de Engenharia Agrícola e Ambiental, Campina Grande, v14, n.4, p.358-365, 2010.

EMPRESA BRASILEIRA DE PESQUISA AGROPECUÁRIA - Centro Nacional de Pesquisas de Solos. Sistema brasileiro de classificação de solos. Rio de Janeiro, 1999. 412p.

EMPRESA BRASILEIRA DE PESQUISA AGROPECUÁRIA - Centro Nacional de Pesquisas de Solos. Manual de métodos de análise do solo. $2^{a}$ Ed. Rio de Janeiro, 1999. 212p.

FONTENELE, W.; SALVIANO, A.A.C.; MOUSINHO, F.E.P. Atributos físicos de um Latossolo Amarelo sob sistemas de manejo no cerrado piauiense. Revista Ciência Agronômica, Fortaleza, v.40, n.2, p194-202, 2009.

ISAAKS, E.H.; SRIVASTAVA, R.M. An introduction to applied geostatistics. New York: Oxford University Press, 1989. 561p.

MARTELLETO, L.A.P.; RIBEIRO, R.D.L.D.; MARTELLETO, M.S.; VASCONCELLOS, M.A.D.S.; MARIN, S.L.D.; PEREIRA, M.B. Cultivo orgânico do mamoeiro 'Baixinho de Santa Amália' em diferentes ambientes de proteção. Revista Brasileira de Fruticultura, Jaboticabal, v.30, n.3, p.662-666, 2008.

MELO, A.A.B.D. Distribuição espacial de alguns atributos de um Argissolo Amarelo sob diferentes manejos no Perímetro irrigado do Baixo Acaraú-CE. 2012. 85f. Dissertação (Mestrado em Agronomia) - Universidade Federal do Ceará, Fortaleza, 2012.

R DEVELOPMENT CORE TEAM. R: A language and environment for statistical computing. R Foundation for Statistical Computing, Vienna, Austria. 2013. Disponível http://www.R-project. org/. Acesso em 16 de Nov 2013.

ROQUE, M.W.; MATSURA, E.E.; SOUZA, Z.D.; BIZARI, D.R.; SOUZA, A.D. Correlação linear e 
espacial entre a resistência do solo ao penetrômetro e a produtividade do feijoeiro irrigado. Revista Brasileira de Ciência do Solo, v.32, n.5, p.18271835, 2008.

SANCHEZ, R.B.; MARQUES JÚNIOR, J.; SOUZA, Z.D.; PEREIRA, G.T.; MARTINS FILHO, M.V. Variabilidade espacial de atributos do solo e de fatores de erosão em diferentes pedoformas. Bragantia, Campinas, v.68, n.4, p.873-884, 2009.

SILVA, E.A. Caracterização e gênese de solos em áreas de depressão de topo de tabuleiros costeiros do nordeste brasileiro. 2012. 99f. Tese (Doutorado em Ciência do Solo) - Universidade Federal de Lavras, Lavras, 2012.

SILVA, J.M.; LIMA, J.S.S.; PIRES, F.R.; ASSIS, R.L. Variabilidade espacial dos atributos físicos em um latossolo sob plantio direto e preparo convencional no cultivo da soja no cerrado. Revista de Ciências Agrárias/Amazonian Journal of Agricultural and Environmental Sciences, v.50, n.1, p.167-180, 2011.

SOARES, S.R.F. Atributos físicos e químicos do solo sob formas de cultivo no assentamento milagre, Apodi/RN. 2013. 32f. Dissertação (Mestrado em Ciência do Solo) - Universidade Federal Rural do Semi-árido, Mossoró, 2013.

SOUZA, J.M. Atributos físico-químicos do solo em lavoura de café conilon submetida à subsolagem. 2013. 75f. Dissertação (Mestrado em Agricultura Tropical) - Universidade Federal do Espírito Santo, São Mateus. 2013.

TAVARES, U.E.; ROLIM, M.M.; PEDROSA, E.M.; MONTENEGRO, A.A.; MAGALHÃES, A.G.; BARRETO, M.T. Variabilidade espacial de atributos físicos e mecânicos de um Argissolo sob cultivo de cana-de-açúcar1. Revista Brasileira de Engenharia Agrícola e Ambiental, Campina Grande, v.16, n.11, p.1206-1214, 2012.

VENDRUSCOLO, J.; RIBEIRO, T.S.; MESQUITA, F.O.; ALVES, A.S.; SANTOS,
G.A.D. Propriedades físicas de um latossolo e argilosso comparados com quatro tipos de solos na paraíba-brasil. Revista Verde de Agroecologia e Desenvolvimento Sustentável, v.6, n.4, p.204212, 2011.

VIEIRA, S.R. Geoestatística em estudos de variabilidade espacial do solo. In: NOVAIS, R.F.; ALVAREZ V., V.H.; SCHAEFER, C.E.G.R. (Eds.). Tópicos em ciência do solo. Viçosa, MG: Sociedade Brasileira de Ciência do Solo, 2000. p.1-54.

VITÓRIA, E.L. Variabilidade espacial da produtividade de plantas forrageiras em diferentes sistemas de manejo do solo. 2010. 114f. Tese (Doutorado em Engenharia Agrícola) Universidade Federal de Viçosa, Viçosa. 2010.

VITORIA, E.L.; FERNANDES, H.C.; TEIXEIRA, M.M. Correlação linear e espacial entre produtividade de capim-mombaça e atributos físicos solo em função do sistema de manejo. Revista Agrotecnologia, Anápolis v.2, n.2, p.30-43, 2012.

ZUCOLOTO, M; LIMA, J.S.S; COELHO, R.I.; SILVA, S.A.; PASSOS, R.R. Variabilidade espacial de atributos fiscos e variáveis agronômicas de citrus em uma parcela experimental em Jerônimo Monteiro (ES). Revista Engenharia na Agricultura, Viçosa, v.19, n.1, p.28-35, 2011.

ZUFFO, V.J. Efeitos de diferentes preparos nos atributos físicos do solo e no desempenho produtivo do mamoeiro Tainung 01. 2012. $85 \mathrm{f}$. Dissertação (Mestrado em Agricultura Tropical) - Universidade Federal do Espírito Santo, São Mateus. 2012.

ZUFFO, V.J.; PIRES, F.R.; BONOMO, R.; VITÓRIA, E.L.; CELIN FILHO, A.; SANTOS, E.O.J. Effects of tillage systems on physical properties of a cohesive yellow argisol in the northern state of Espírito Santo, Brazil. Revista Brasileira de Ciência do Solo, Viçosa, v.37, n.1, p.1372-1382, 2013. 
WALTER, A.M.; CHRISTENSEN, S.; WARRICK, A.W.; NIELSEN, D.R. Spatial SIMMELSGAARD, S.E. Spatial correlation variability of soil physical properties in the field. between weed species densities and soil properties. In: HILLEL D. (Ed.). Aplicattions of soil physics, Weed Res., v.42, p.26-38, 2002. New York, v.2, p319-344, 1980 